\title{
Role of Multidetector Computed Tomography in Evaluation of Renal Infections \\ Hayam A. Abdellatif, Hager S. Sakr, El-Sayed M. El-Sayed
}

Radiodiagnosis Department, Faculty of Medicine, Menoufia University, Menoufia, Egypt

*Corresponding Author: Hager Sobhy Ali Sakr, Mobile: 00201000888162, E-mail: googalygogo@ gmail.com

\begin{abstract}
Background: Imaging plays a very important role in the diagnosis of renal infection. It assists diagnosis and differentiates between different types of renal infection. It also helps to detect and manage the associated complications through image-guided drainage and aspiration. Contrast-enhanced CT is the most useful study. A contrast-enhanced study is essential for a complete evaluation of patients with renal inflammatory disease to demonstrate alterations in the renal parenchymal perfusion and excretion of the contrast material, which occur as a result of the inflammatory process. Objective: To detect the role of multidetector CT in diagnosis of renal infection. Materials and methods: This prospective study was conducted on sixty patients. The study was carried out at Diagnostic Radiology Departments of Menoufia University Hospital. The patients were presenting with fever (> $38^{\circ} \mathrm{C}$ ) mainly and loin pain. Results: From 60 patients with renal infection, 18 cases were diagnosed as pyonephrosis, 16 cases were diagnosed as renal abscess, 12 cases were diagnosed as pyelonephritis, 4 cases were diagnosed as xanthogranulomatous pyelonephritis, 4 cases were diagnosed as perinephric abscess, 2 cases were diagnosed as emphysematous pyelonephritis, 2 cases were diagnosed as fungal ball, 2 cases were diagnosed as urinoma. The mean age was $(39.4 \pm 15.7)$.
\end{abstract}

Conclusion: Contrast enhanced computed tomography is a perfect diagnostic modality to assess the severity of inflammatory process and reaching the diagnosis of renal infection. It also helps to reach the cause, predisposing factors, associations, complications and the extent of the disease.

Keywords: Abdominal computed tomography, Inflammation, Renal infection.

\section{INTRODUCTION}

Renal infections include acute pyelonephritis, renal abscess, perinephric abscess, emphysematous pyelonephritis, xanthogranulomatous pyelonephritis, and renal tuberculosis. Renal infections are generally well evaluated with multidetector CT (MDCT). Although CT is not routinely indicated in uncomplicated renal infection, it is important in establishing the diagnosis in equivocal cases, in evaluating high-risk patients and in determining the extent of disease ${ }^{\mathbf{( 1 )}}$.

Contrast material-enhanced study is essential for complete evaluation of patients with renal infections to demonstrate alterations in renal excretion of contrast material that occurs as a result of the inflammatory process. Delayed CT scans obtained during the excretory phase are frequently more helpful than early CT scans in defining the extent of the disease process, and identifying its complications such as abscess formation, xanthogranulomatous pyelonephritis and urinary obstruction (2). Acute renal infections span a spectrum of varying severity from uncomplicated acute pyelonephritis through progressively worsening stages of inflammation to frank abscess formation ${ }^{(3)}$. Because histologic specimens are difficult to obtain, exact clinical correlation with renal imaging is to provide information regarding the nature and extent of the disease $^{(\mathbf{1})}$.

CT is the mainstay of diagnostic imaging for xanthogranulomatous pyelonephritis as it allows a confident diagnosis and gives information about the extra renal extent of the disease ${ }^{(4)}$. CT has major role in assessment of renal tuberculosis; it provides an accurate evaluation of amount of residual functioning parenchyma and extra renal spread and also early manifestations as calyceal erosion or papillary necrosis ${ }^{(5)}$. CT scanning is the diagnostic modality of choice in assessment of perinephric abscess because it is more sensitive, accurate in defining the precise location, size, degree, and extent of the loculation in relation to other retroperitoneal structures ${ }^{\left({ }^{(}\right)}$. CT has an important role in early detection of the underlying abnormalities as well as associated complications such as abscess. In addition, it may detect associated pathologies such as renal calculi and diverticula ${ }^{(7)}$.

Aim of the present study was to detect the role of multidetector CT in diagnosis of renal infection.

\section{PATIENTS AND METHODS}

We prospectively evaluated 60 patients with renal infection diagnosed by clinical and preliminary imaging modalities, who were referred to the Radiology Department in Menoufia University from (Urology, Nephrology and other departments). The patient came to the hospital underwent:1) Clinical history and examination e.g. fever and loin pain, 2) Laboratory investigations e.g. complete blood culture(CBC) and urine analysis, and 3) Ultrasonography, then the patient underwent contrast enhanced CT examination after obtaining detailed clinical history, explanation of the procedure and getting informed consent in written form. The patient was scanned within the Radiology Unit by Toshiba Alexion a 16-multi-slice, Japan with the subsequent parameters: helical acquisition, $120 \mathrm{kV}$, $25 \mathrm{~mA}$, helical thickness $10 \mathrm{~mm}, 1 \mathrm{~mm}$ and FOV35 $\mathrm{cm}$ 
starting just above the higher dome of diaphragm to the iliac crest, patients were lying supine in feet first position at complete rest, hands were placed behind head. All instructions were given to the patient about table movements, voice massages, and sensation of contrast injection, timing and manner of breath holding. Non contrast scan: is necessary as a baseline to assess the enhancement. Post contrast scan :approximately 90$100 \mathrm{ml}$ of a nonionic contrast material (omnipaque) was injected at a rate of 3-4 ml/sec through a 16/18-gauge cannula placed in an antecubital vein, the following phases were taken :a) corticomedullary phase: the cortex enhances more rapidly than the medulla, appearing more dense from around $20 \mathrm{~s}$ to $60 \mathrm{~s}$ when there is maximum corticomedullary differentiation, b) nephrographic phase: At approximately 100 to 120 seconds after contrast injection, enhancement of the cortex and medulla equilibrates, and c) excretory phase scanning: After at least 3 minutes after injection, excretion from the renal tubules begins to fill the renal calyces and renal pelvis. Axial, sagittal and coronal cuts were taken and AP scan projection radiograph was obtained.

Inclusion criteria: High fever $\left(>38^{\circ} \mathrm{C}\right)$, abdominal or loin pain, leukocytosis count exceeding $11,000 / \mathrm{mm}^{3}$, patients with 2 or more of these findings were included in the study.

Exclusion Criteria: Pregnant and lactating patients, children, Cardiac failure, previous allergic reaction to contrast media, other febrile disease such as colitis, pneumonia, acute prostatitis, acute epididymitis, or pelvic inflammatory disease.

\section{Ethical considerations:}

Approval from Research Ethics committee (REC) of Menoufia University was taken before starting fieldwork. All the procedures of the study were approved by the Diagnostic Radiology Department. Agreement of participant without obligation was taken. Confidentiality of data was preserved. Explanation of this project to the participants was done. An informed written permission was taken from the patients to contribute in the study, after clarifying the aim of the study.

\section{Statistical analysis}

Data were collected, tabulated and statistically analyzed using an IBM compatible personal computer with Statistical Package for the Social Sciences (SPSS) version 23 (SPSS Inc. Released 2015. IBM SPSS statistics for windows, version 23.0, Armnok, NY: IBM Corp.). Qualitative data were expressed in: number and percentage, while quantitative data were expressed as mean, standard deviation and range (minimummaximum). Fischer's exact test was used to study association between qualitative variables. Significant test results were quoted as two-tailed probabilities. Significance of the obtained results was judged at the $5 \%$ level $(\mathrm{P}>0.05)$.

\section{RESULTS}

In our study 56 cases had leukocytosis. 50 cases had positive urine culture as shown in Table (1).

Table (1): Laboratory results of participants $(\mathrm{N}=60)$

\begin{tabular}{|l|c|c|}
\hline \multirow{2}{*}{ Laboratory results } & \multicolumn{2}{|c|}{ Frequency } \\
\cline { 2 - 3 } & N & \% \\
\hline WBCs: & & \\
Normal & 4 & 6.7 \\
Leucocytosis $\left(>11,000 / \mathrm{mm}^{3}\right)$ & 56 & 97.3 \\
\hline Urine culture: & & \\
Positive & 50 & 83.3 \\
Negative & 10 & 16.7 \\
\hline
\end{tabular}

The details of CT findings are shown in table (2).

Table (2): CT findings of the patients $(\mathrm{N}=60)$

\begin{tabular}{|c|c|c|}
\hline \multirow[t]{2}{*}{ CT findings } & \multicolumn{2}{|c|}{ Frequency } \\
\hline & $\mathbf{N}$ & $\%$ \\
\hline Lesion: & & \\
\hline Focal & 18 & 30 \\
\hline Diffuse & 42 & 70 \\
\hline Size of focal lesions $(\mathrm{cm})$ : & \multirow{3}{*}{\multicolumn{2}{|c|}{$\begin{array}{c}5.2 \pm 1.4 \\
3-7\end{array}$}} \\
\hline Mean \pm SD & & \\
\hline Range & & \\
\hline \multicolumn{3}{|l|}{ Site: } \\
\hline Left kidney & 24 & 40 \\
\hline Right kidney & 30 & 50 \\
\hline Bilateral & 6 & 10 \\
\hline \multicolumn{3}{|l|}{ Margin: } \\
\hline Ill defined & 40 & 66.7 \\
\hline Well defined & 20 & 33.3 \\
\hline \multicolumn{3}{|l|}{ Attenuation: } \\
\hline High & 22 & 36.7 \\
\hline Intermediate & 4 & 6.7 \\
\hline Low & 28 & 46.7 \\
\hline Mixed & 6 & 10 \\
\hline \multicolumn{3}{|l|}{ Pattern of enhancement: } \\
\hline Heterogenous & 18 & 30 \\
\hline Homogenous & 2 & 3.3 \\
\hline Ring enhancement & 14 & 23.3 \\
\hline Striated nephrogram & 4 & 6.7 \\
\hline No & 22 & 36.7 \\
\hline \multicolumn{3}{|l|}{ Containing air lesion: } \\
\hline Negative & 42 & 70 \\
\hline Positive & 18 & 30 \\
\hline \multicolumn{3}{|l|}{ Fat stranding: } \\
\hline Negative & 18 & 30 \\
\hline Positive & 42 & 70 \\
\hline \multicolumn{3}{|l|}{ Associations: } \\
\hline No & 20 & 33.3 \\
\hline Stones & 18 & 30 \\
\hline Renal abscess & 4 & 6.7 \\
\hline Psoas abscess & 6 & 10 \\
\hline Others & 12 & 20 \\
\hline
\end{tabular}

As regards to the number of cases that were diagnosed by CT and US, we could reach the diagnosis by $\mathrm{CT}$ in 48 while regarding US we could reach the 
diagnosis in 24 cases. The details of the different diagnoses are shown in table (3).

Table (3): Diagnosis of the participant cases $(\mathrm{N}=60)$

\begin{tabular}{|l|c|c|}
\hline Diagnosis & \multicolumn{2}{|c|}{ Frequency } \\
\cline { 2 - 3 } & $\mathbf{N}$ & $\mathbf{\%}$ \\
\hline Diagnosis by CT: & 12 & 20 \\
Negative & 48 & 80 \\
Positive & & \\
Diagnosis by US: & 36 & 60 \\
Negative & 24 & 40 \\
Positive & & \\
\hline Diagnosis of negative cases in both CT and US: & 8 & 13.3 \\
Biopsy proven & 52 & 86.6 \\
No & & \\
Final diagnosis: & 16 & 26.7 \\
Renal abscess & 18 & 30 \\
Pyonephrosis & 12 & 20 \\
Pyelonephritis & 4 & 6.7 \\
Xanthogranulomatous pyelonephritis & 2 & 3.3 \\
Emphysematous pyelonephritis & 4 & 6.7 \\
Perinephric abscess & 2 & 3.3 \\
Fungal ball & 2 & 3.3 \\
Urinoma & & \\
Confirmation of diagnosis: & 22 & 36.7 \\
Percutaneous drainage of pus & 8 & 13.2 \\
Biopsy proven & 4 & 6.7 \\
Endoscopic drainage of pus & 10 & 16.7 \\
Surgical drainage of pus & 16 & 26.7 \\
No & & \\
\hline
\end{tabular}

In our study the sensitivity of CT in diagnosis of renal infections was $83 \%$, with specificity $22 \%$, accuracy $47 \%$, PPV 42\%, NPV 67\% (Table 4).

Table (4): Diagnostic accuracy of CT in renal infections $(n=60)$

\begin{tabular}{|c|c|c|c|c|c|c|}
\hline \multirow{3}{*}{$\begin{array}{l}\text { CT in } \\
\text { renal } \\
\text { infections }\end{array}$} & \multicolumn{4}{|c|}{ US in renal infections } & \multirow{3}{*}{$\begin{array}{l}\text { Fisher's } \\
\text { exact test }\end{array}$} & \multirow{3}{*}{ P-value } \\
\hline & \multicolumn{2}{|c|}{$\begin{array}{c}\begin{array}{c}\text { Positive } \\
(n=24)\end{array} \\
\end{array}$} & \multicolumn{2}{|c|}{$\begin{array}{c}\text { Negative } \\
\text { (36) }\end{array}$} & & \\
\hline & $\mathbf{N}$ & $\%$ & $\mathbf{N}$ & $\%$ & & \\
\hline Positive & 20 & 83.3 & 28 & 77.8 & \multirow[b]{2}{*}{0.3} & \multirow[b]{2}{*}{0.427} \\
\hline Negative & 4 & 16.7 & 8 & 22.2 & & \\
\hline Sensitivity & \multicolumn{6}{|c|}{$83 \%$} \\
\hline Specificity & \multicolumn{6}{|c|}{$22 \%$} \\
\hline Accuracy & \multicolumn{6}{|c|}{$47 \%$} \\
\hline PPV & \multicolumn{6}{|c|}{$42 \%$} \\
\hline NPV & \multicolumn{6}{|c|}{$67 \%$} \\
\hline
\end{tabular}

PPV: positive predictive value, NPV: Negative predictive value.

The sensitivity, specificity, accuracy, positive predictive value (PPV), negative predictive value (NPV) of CT in diagnosis of renal abscess, pyonephrosis, and pyelonephritis (Figure 1). 


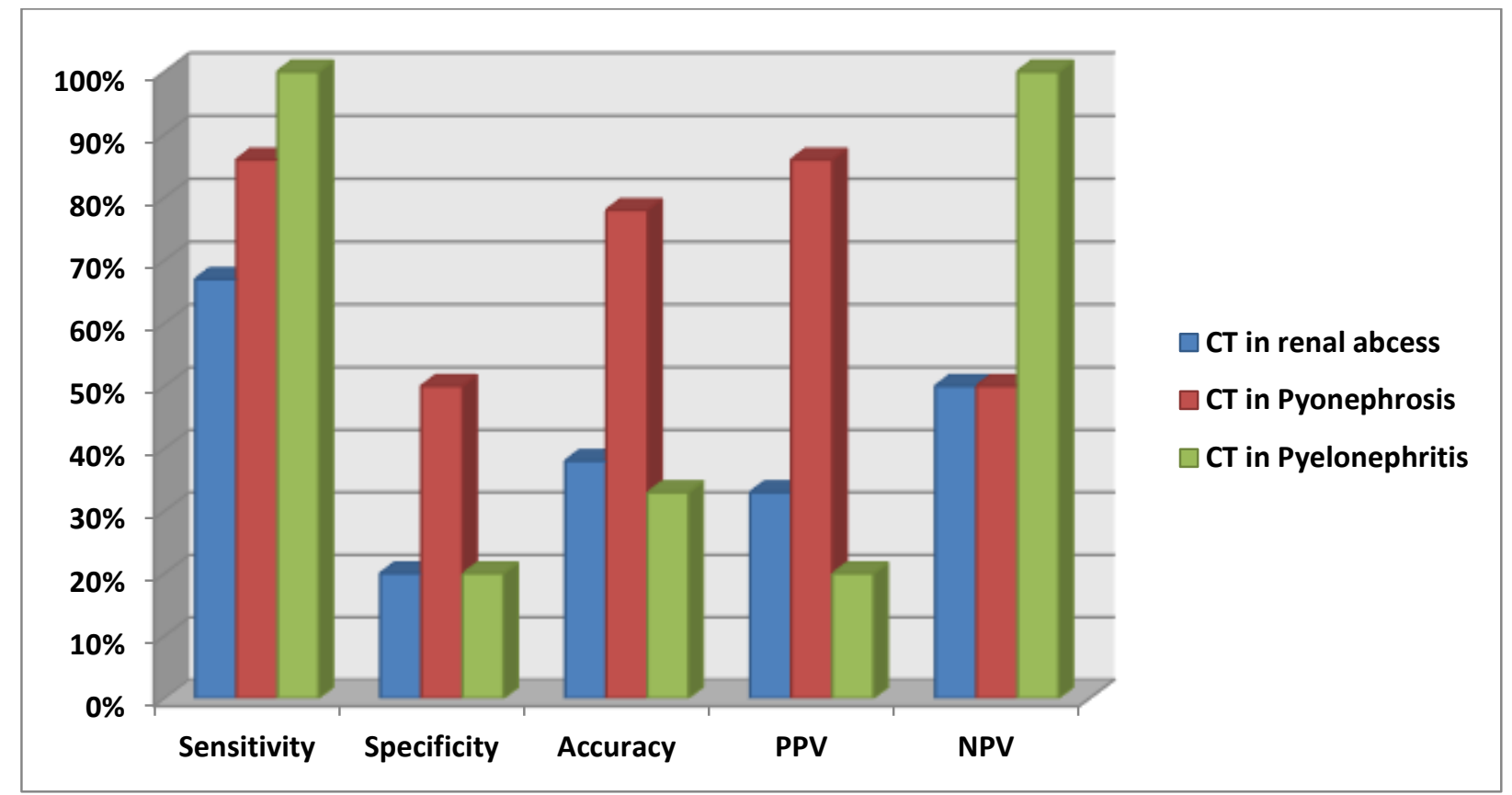

Figure (1): Diagnostic accuracy of CT in renal abscess, pyonephrosis and pyelonephritis

A case of right sided pyonephrosis with lower calyceal stones.

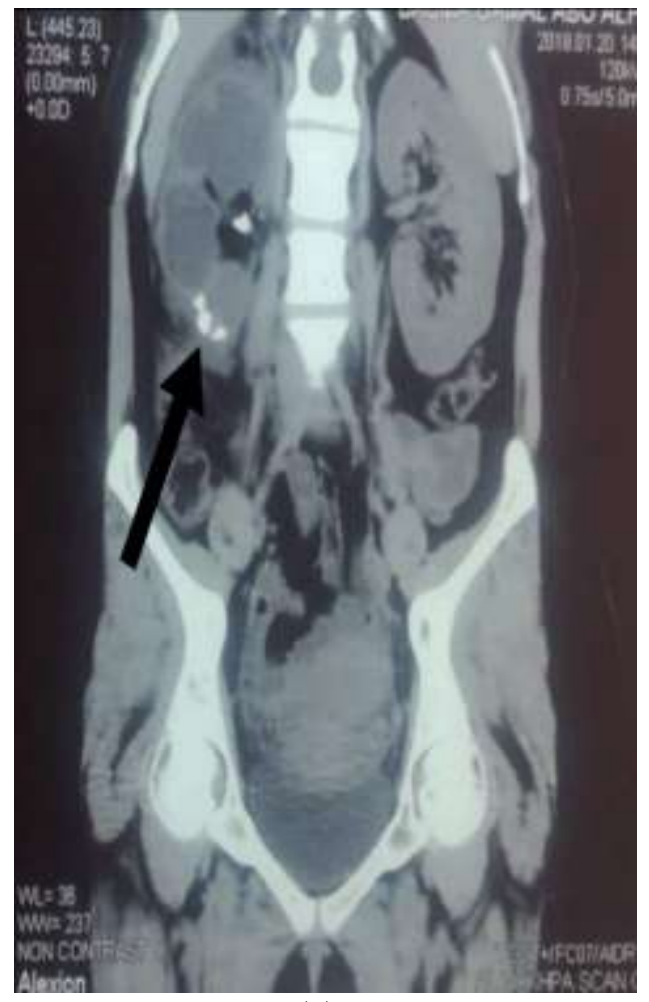

(a)

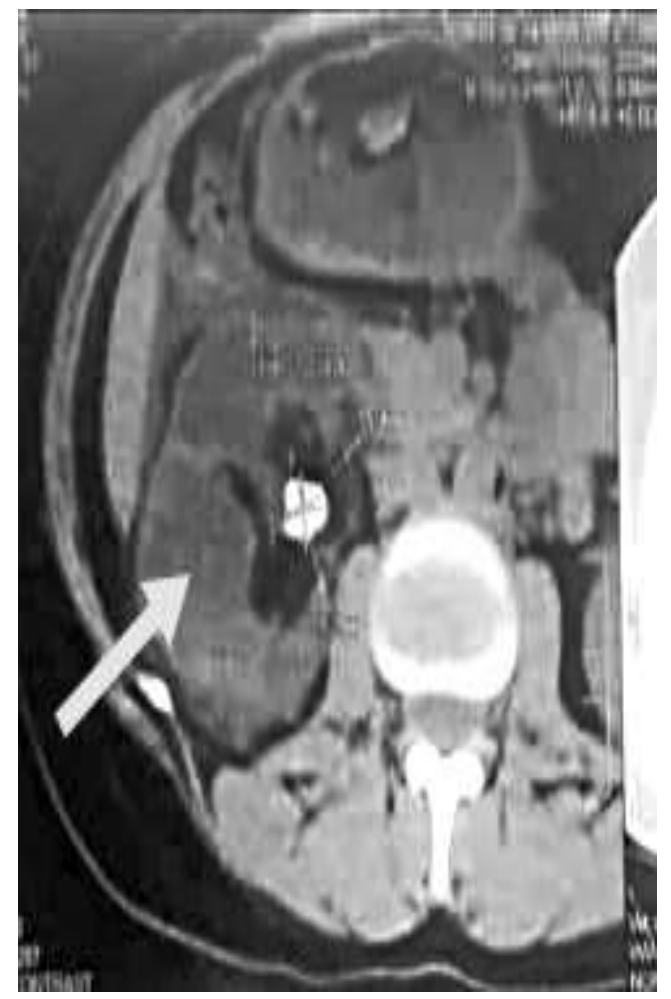

(b)

Figure (2): Female patient 46 years old presented with fever $\left(>38^{\circ} \mathrm{C}\right)$ lasting for 7 days and right loin pain with leucocytosis and positive urine culture, abdominal ultrasound revealed hydronephrosis with debris. (a) CT coronal image shows the right kidney is diffusely enlarged with mild dilatation of the collecting system with high attenuation value of fluid collection within it, also pelvic wall thickening is noted with perinephric fat stranding, and also there is multiple small stones in the lower calyces (black arrow). (b) CT axial non contrast image showing dilatation of the collecting system with higher attenuation values of fluid within the renal collecting system (white arrow) with thickening of the pelvic walls. 


\section{A case of left sided renal abscess}
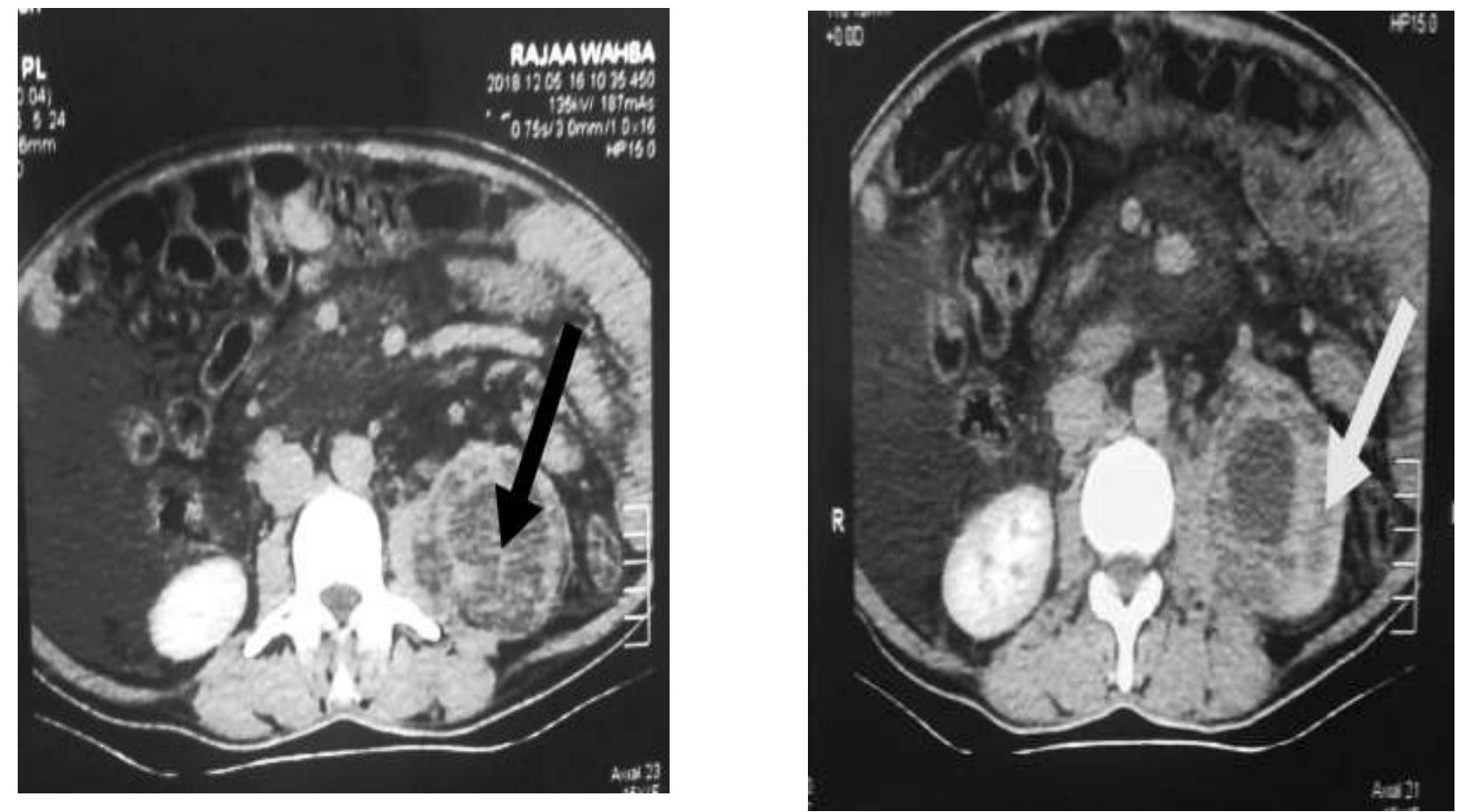

(a)

(b)

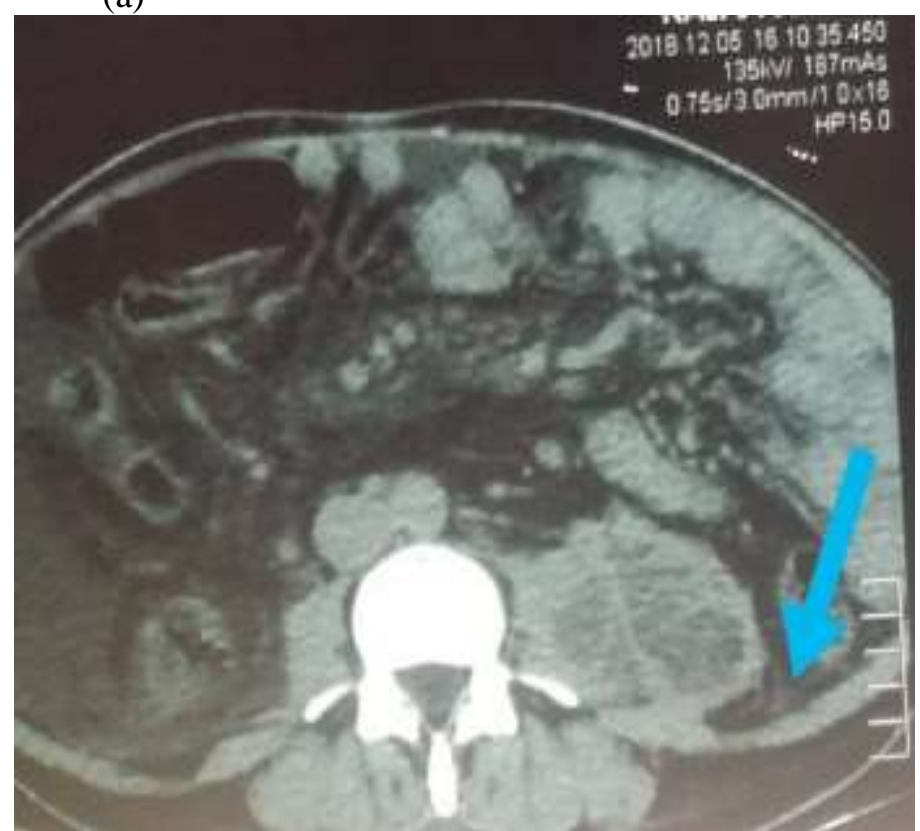

(c)

Figure (3): Female patient 38 years old presented with fever $\left(>38^{\circ} \mathrm{C}\right)$ lasting for 9 days, loin pain and abdominal swelling with leucocytosis and positive urine culture. $(a, b, c)$ CT axial cuts post contrast at the nephrogenic phase showing the left kidney is diffusely enlarged with multilocular cystic hypodense large lesion in the renal parenchyma (black arrow in a) with ring enhancement at the left kidney (white arrow in b) indicating enhancement of the rim of pseudocapsule and perinephric fat stranding (blue arrow in c). 


\section{A case of xanthogranulomatous pyelonephritis of the right kidney}

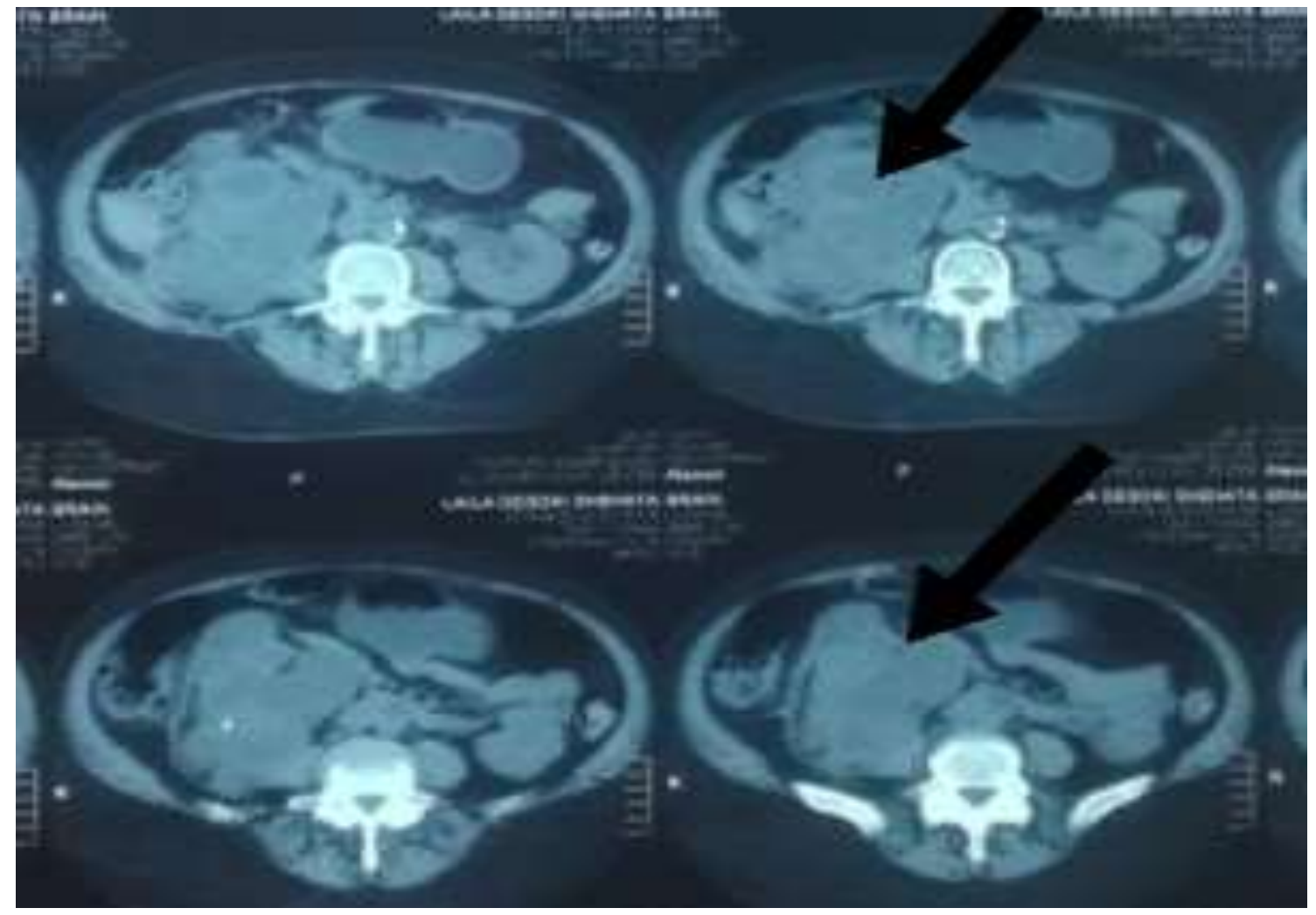

(a)

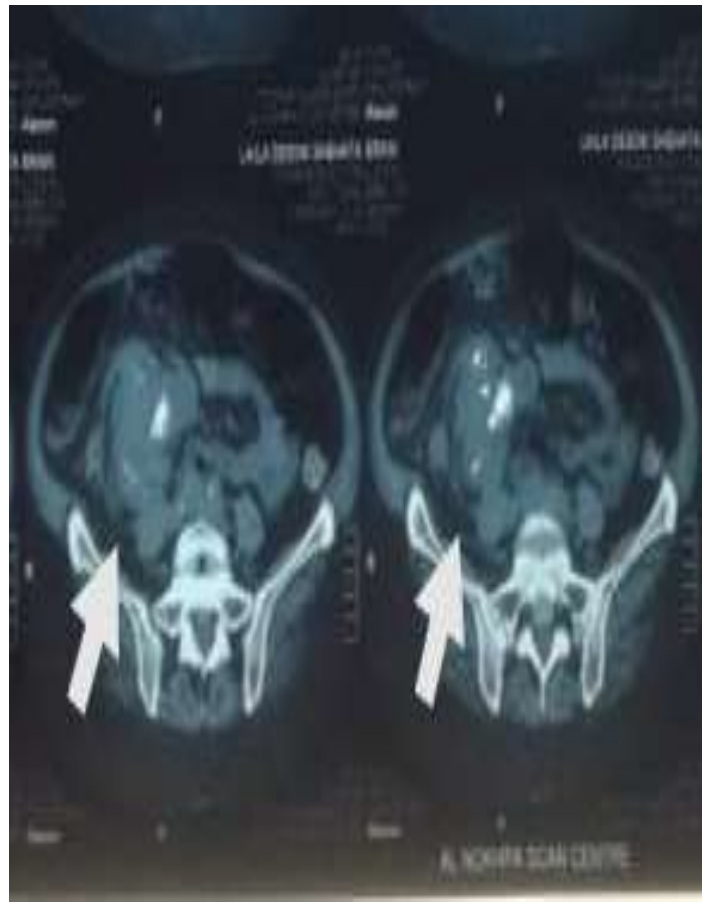

(b)

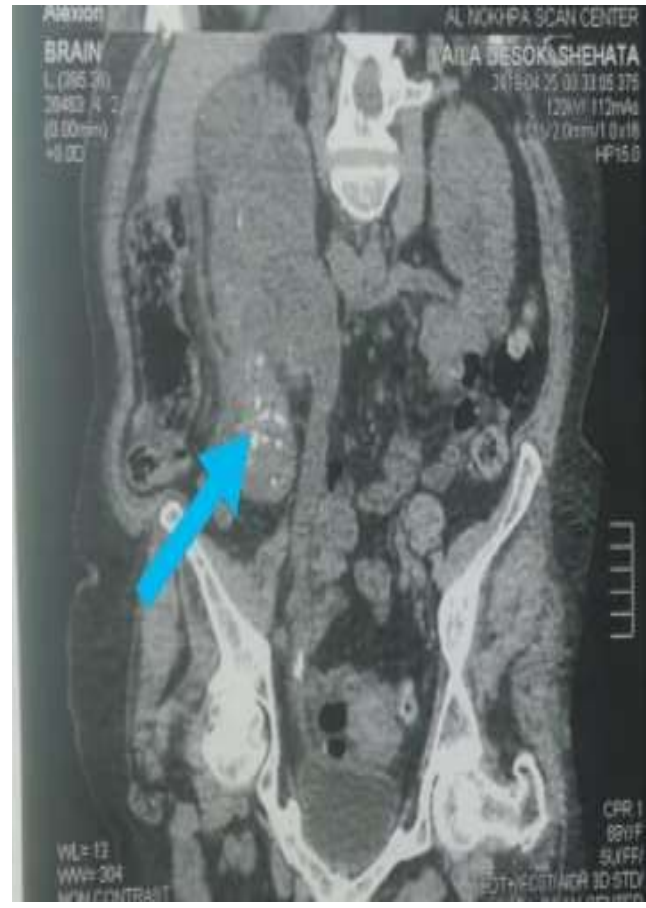

(c)

Figure (4): Female patient 22 years old presented with fever $\left(>38^{\circ} \mathrm{C}\right)$ lasting for 12 days, hematuria and loin pain with leucocytosis and positive urine culture. $(\mathrm{a}, \mathrm{b}) \mathrm{CT}$ axial cuts showing the right kidney is markedly enlarged in size showing lobulated outline and loss of corticomedullary differentiation (black arrow in a) associated with thickened gerota fascia and stranding of perinephric fat (white arrow in b). Multiple lower calyceal renal stones are noted (blue arrow in c); the largest measures $22 X 16 \mathrm{~mm}$ showing an attenuation value of 87HU. (c) CT coronal cut showing the right kidney is enlarged fat density was measured with stranding of perinephric fat with multiple lower calyceal stones (blue arrow) and multiple lower ureteric stones; the largest measures 7X10 mm showing an attenuation value of $397 \mathrm{HU}$. 


\section{A case of left renal fungal ball}

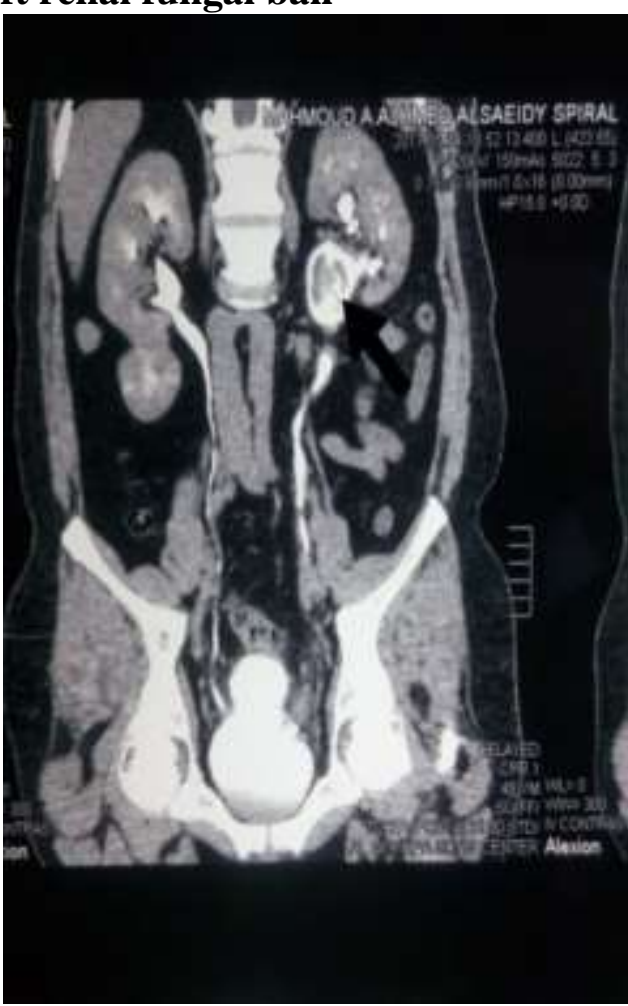

(a)

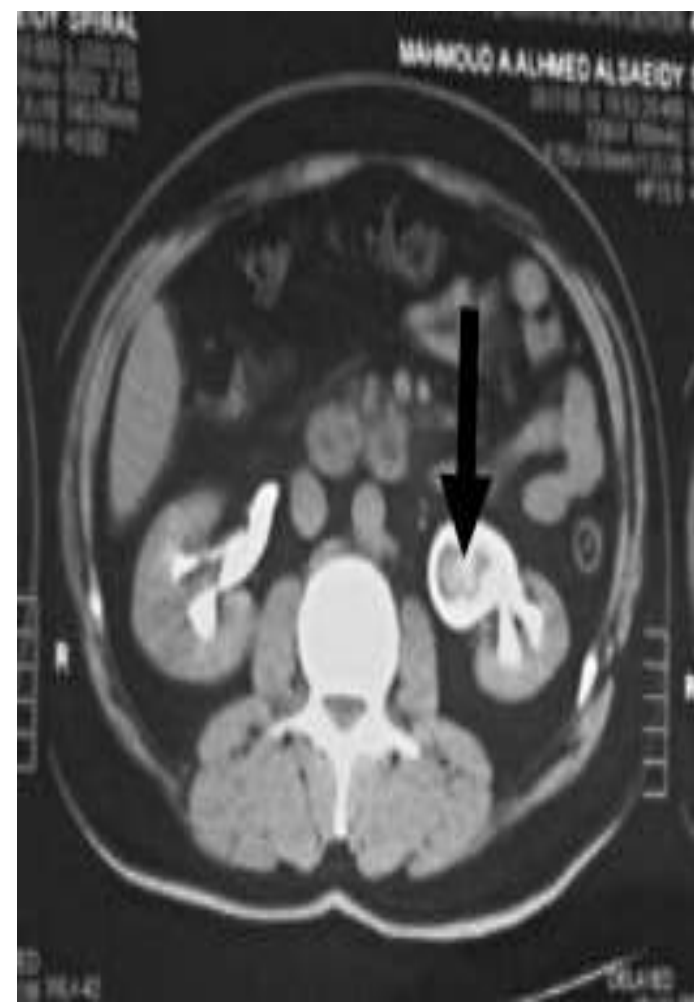

(b)

Figure (5): Male patient 20 years old presented with hematuria and loin pain with past history of ureteroscopic operation for a ureteric stone 5 months ago with leucocytosis and negative urine culture. (a , b) CT coronal and axial cuts post contrast at execratory phase showing left renal isodense mass with filing defect surrounding the ureteric catheter (black arrow) with mild hydroureteronephrosis, the mass shows no enhancement with wall enhancement of the ureter is seen in delayed coronal reconstructed image shows contrast-filled urine all around the lesion with dilated pelvis and caliectasis.

\section{A case of bilateral emphysematous pyelonephritis.}
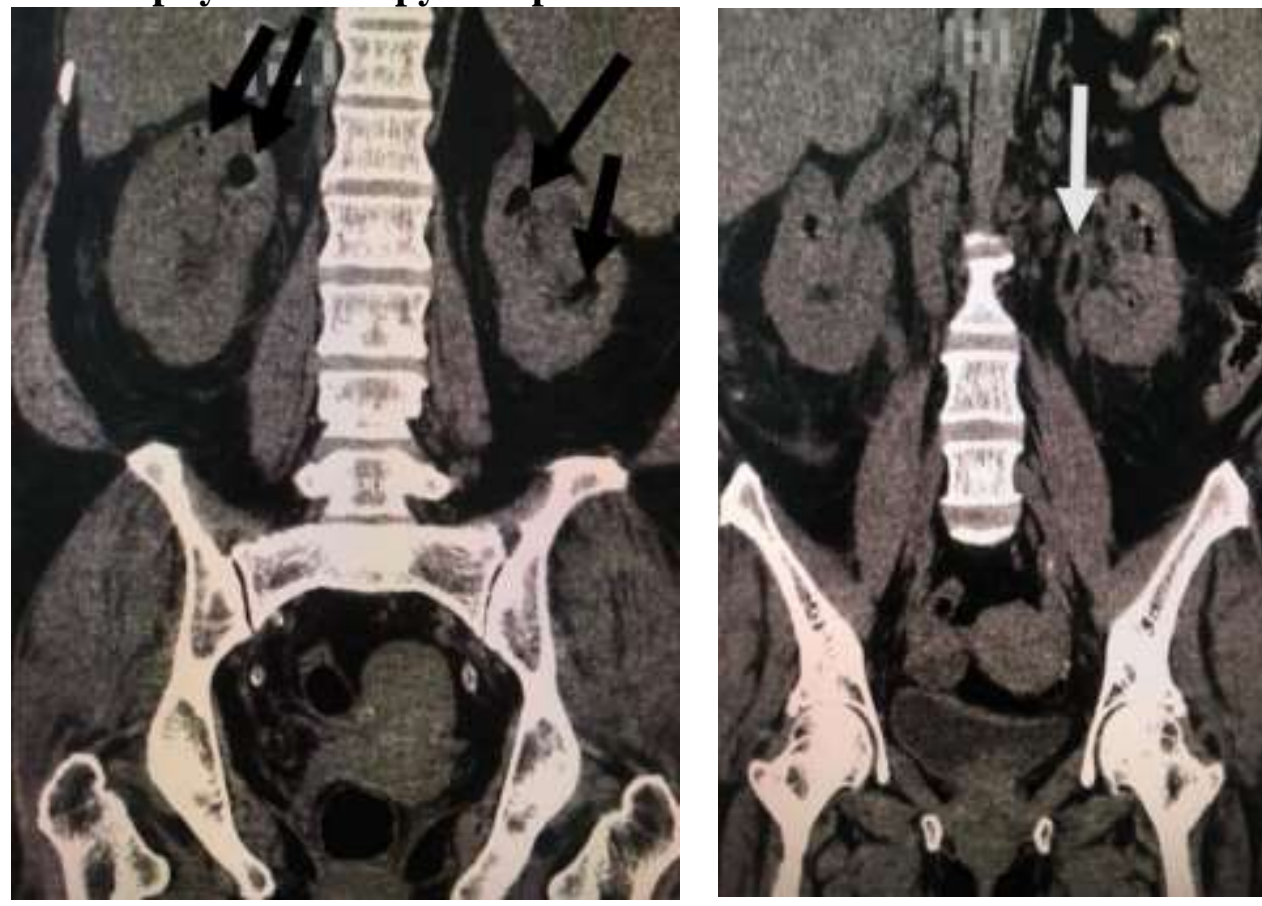

(a)

(b)

Figure (6): Female patient 22 years old presented with fever $\left(>38^{\circ} \mathrm{C}\right)$, loin pain and toxaemia with leucocytosis and positive urine culture. (a, b) CT coronal cuts without contrast showing bilateral linear and mottled streaks of gas in bilateral renal cortex and left collecting system (black arrow in a) and left upper third of ureter (white arrow in b). 


\section{DISCISSION}

MDCT is more useful than intravenous urography (IVU) or ultrasonography for the evaluation of renal infection. MDCT urography (MDCTU) has allowed radiologists to overcome most of the limitations of spectral detector CT (SDCT). Particularly, the high speed of acquisition, the thinslice collimation, and the near isotropy of the voxels allow the reformatting of images in any plane without significant artifacts and with excellent anatomic detail.

In this study, the age of patients ranged from 5 to 65 years old with the mean age $(39.4 \pm 15.7)$. The study showed prevalence of renal infections in females $(63.3 \%)$ in comparison to males $(36.7 \%)$. This goes with Albracht et al. ${ }^{(8)}$ who stated that Urinary tract infections (UTIs) are generally considered a disease of women. However, UTIs affect females throughout the lifespan, and certain male populations (including infants and elderly men) are also susceptible. Epidemiologically, pyelonephritis is more common in women but carries increased morbidity when it does occur in men.

In this study, the nephrolithiasis was the most common predisposing factor $(n=18)$ followed by diabetes mellitus $(n=12)$ then medical instruments representing $(n=8)$ and anatomical abnormalities $(n=6)$ and immunocompromised patients $(n=4)$ also recurrent UTI $(n=4)$. This goes with Soulen et al. ${ }^{(9)}$ who stated that factors predisposing to renal infection were present in two-thirds of the patients. The most common were pre-existing urinary tract disease, including nephrolithiasis $(n=16)$, recurrent urinary tract infections $(n=12)$, anatomic abnormalities of the urinary tract $(n=5)$, and neuropathic bladder $(n=4)$. Four of the 39 patients $(10 \%)$ had diabetes mellitus.

Regarding the complaint: fever more than $38^{\circ} \mathrm{C}$ was the main complaint in the whole 60 cases of renal infection lasting for several days with mean \pm SD $(8.4 \pm 2.8)$ and median 8). In combination with fever, 48 cases were complaining also of loin pain and 10 cases were complaining of loin pain and hematuria, while only 2 cases were complaining of toxaemia and also 56 cases had leucocytosis $>11,000 / \mathrm{mm}^{3}$, while 4 cases had normal WBCs count. Regarding urine analysis 50 cases had positive urine culture while 10 cases had negative culture .This goes with Stunell $\boldsymbol{e t}$ al. (10) who stated that the diagnosis is based on a combination of typical clinical features and laboratory findings. Typical clinical features include urinary frequency, dysuria, flank pain and a high grade fever $\left(>38.5^{\circ} \mathrm{C}\right.$ ) accompanied by rigors. Laboratory findings include pyuria, white cell casts, bacteriuria and a positive urine culture. In addition, acute-phase reactants including erythrocyte sedimentation rate (ESR), C-reactive protein (CRP) and blood white cell count are often elevated. Bacteraemia and sepsis are common, especially in high-risk patient groups. This also goes with Rollino et al. (11) who stated that leucocytosis was evident in 183 patients $(82.06 \%)$; mean leucocytes of these patients were $16960 \pm 5869$ / $\mathrm{mm}^{3}$. Leucocytosis normalized in $4.21 \pm 3.73$ days. Mean CRP was $15.65 \pm 8.56 \mathrm{mg} / \mathrm{dL}$. Pyuria was present in 147 patients $(65.92 \%)$.

In our study 42 lesions were diffuse while only 18 lesions were focal with mean \pm SD size of $5.2 \pm 1.4$. In correlation to the attenuation of the lesion in CT, 28 lesions had low attenuation and 22 lesions hade high attenuation while 6 lesions showed mixed attenuation and 4 lesions had intermediate attenuation, regarding the pattern of enhancement 18 lesions showed heterogenous enhancement and 14 lesions showed ring enhancement while only 4 cases had striated nephrogram and 2 cases presented with homogenous enhancement. This goes with Soulen $\boldsymbol{e t}$ al. ${ }^{(9)}$ who stated that Global enlargement of the kidney was present in seven of the ten cases of diffuse acute bacterial nephritis (ABN) but in only one of the six cases of focal ABN. Four of the 16 patients with $A B N$ had inflammatory changes in the perirenal fat, and three had thickening of the Gerota fascia. Contrast material administration demonstrated an ill-defined, inhomogeneous pattern of decreased renal parenchymal enhancement in 12 patients and wedgeshaped segments of striated parenchymal opacification in five patients (one patient had both) (Fig 3). Unenhanced scans obtained in six of these patients showed only subtle foci of decreased attenuation in two cases and were normal in four.

Regarding the 16 cases that were diagnosed as renal abscess, it was found that the sensitivity of CT was $67 \%$ and specificity was $20 \%$. CT was more sensitive than US in detection of renal abscess. This goes with Angel et al. ${ }^{(12)}$ who reported that sonograms and CT scans permit $82-90 \%$ accuracy in the diagnosis of renal and perirenal abscesses. CT scans are most accurate for diagnosis and have the added advantage of distinguishing between renal and perirenal abscesses.

Regarding the 12 cases that were diagnosed as pyelonephritis it was found that the a sensitivity of CT in pyelonephritis was $100 \%$, specificity 20\%, accuracy 33\%, PPV 20\% and NPV 100\% but ultrasound was less sensitive than CT in detection of pyelonephritis. This goes with Mitterberger et al. (13) who found that on contrast CT, 84 patients (84\%) had renal parenchymal changes suggestive of acute pyelonephritis (APN); on contrast US, 82 of the 84 (98\%) showed renal parenchymal changes, and APN was correctly diagnosed. The sensitivity of contrast enhanced CT was 98\%, a specificity of $100 \%$, a positive predictive value of $100 \%$, and a negative predictive value of $89 \%$, which was similar to contrast US. Renal ultrasonography was less sensitive than CT. This also goes with Majd et al. ${ }^{(14)}$ who found that histopathologic examination showed evidence of single or multiple foci of acute pyelonephritis in 38 of 70 kidneys and in 102 of 210 renal zones. The sensitivity and specificity of the imaging modalities for the 
detection of affected kidneys were 93.5\% (101 of 108) for spiral CT, and $56.6 \%$ for power Doppler US.

Considering the 18 cases that were diagnosed as pyonephrosis it was found that sensitivity of CT in detection of pyonephrosis was $86 \%$, specificity $50 \%$, accuracy $78 \%$, PPV86\% and NPP $50 \%$, also US was highly sensitive in detection of pyonephrosis with the same ratio of CT (86\%). This goes with Yuruk et al. ${ }^{(15)}$ who revealed that a HU value of 20 or more was a significant predictor of infection with a sensitivity of $83.4 \%$ and a specificity of $39.3 \%$. Regarding the sensitivity of ultrasound it goes with Kao and Wu ${ }^{(16)}$ where sonography had a sensitivity of $90 \%$ and an overall accuracy of $96 \%$ in the diagnosis of pyonephrosis. Sonographic diagnosis of pyonephrosis depends on the presence of tissue and cellular debris, which produce internal echoes due to acoustic impedance mismatches.

\section{CONCLUSION}

MDCT is highly sensitive in detection of renal infections. It also help to reach the cause, predisposing factors, associations and complications. It also helps to assess the extent of the disease. Treatment planning is based on severity of renal infection and presence of complications. MDCT helps guide management and monitor the success of treatment by follow up.

\section{REFRENCES}

1. Kawashima A, Sandler C, Goldman S (2000): Imaging in acute renal infection. BJU International, 86(1):70-79.

2. Nicolau C, Aldecoa I, Bunesch L et al. (2015): The role of contrast agents in the diagnosis of renal diseases. Current Problems in Diagnostic Radiology, 44(4):346359.

3. Kawashima A, LeRoy A (2003): Radiologic evaluation of patients with renal infections. Infectious Disease Clinics of North America, 17(2): 433-456.

4. Loffroy R, Guiu B, Watfa J et al. (2007): Xanthogranulomatous pyelonephritis in adults: clinical and radiological findings in diffuse and focal forms. Clinical Radiology, 62(9):884-890.

5. Quaia E, Martingano P, Cavallaro M et al. (2011): Multislice CT urography characterizes renal TB. https://www.diagnosticimaging.com/ view/multislicect-urography-characterizes-renal-tb

6. Gardiner R, Gwynne R, Roberts S (2011): Perinephric abscess. BJU International, 107(3):20-23.

7. Browne $\mathbf{R}$, Zwirewich $\mathbf{C}$ (2004): Imaging of urinary tract infection in the adult. European Radiology Supplements, 14(3): 1-1.

8. Albracht C, Hreha T, Hunstad D (2020): Sex effects in pyelonephritis. https://www.X-mol.com/paper/ 1235640737819508736

9. Soulen M, Fishman E, Gatewood O (2000): Bacterial renal infection: role of CT. Radiology, 171(3): 703-707.

10. Stunell H, Buckley O, Feeney J et al. (2006): Imaging of acute pyelonephritis in the adult. European Radiology, 17(7): 1820-1828.

11. Rollino C, Beltrame G, Ferro M et al. (2012): Acute pyelonephritis in adults: a case series of 223 patients. Nephrology Dialysis Transplantation, 27(9): 34883493.

12. Angel C, Shu T, Green J et al. (2003): Renal and perirenal abscesses in children. Pediatric Nephrology, 140(3): 517-520.

13. Mitterberger M, Pinggera G, Colleselli $D$ et al. (2008): Acute pyelonephritis: comparison of diagnosis with computed tomography and contrast-enhanced ultrasonography. BJU International, 101(3): 341-344.

14. Majd M, Blask N, Markle B et al. (2001): Acute pyelonephritis: Comparison of diagnosis with $99 \mathrm{mTc}$ DMSA SPECT, Spiral CT, MR imaging, and power doppler US in an experimental pig model. Radiology, 218(1): 101-108.

15. Yuruk E, Tuken M, Sulejman $S$ et al. (2016). Computerized tomography attenuation values can be used to differentiate hydronephrosis from pyonephrosis. World Journal of Urology, 35(3): 437-442.

16. Kao H, Wu C (2008): Ultrasound of Renal Infectious Disease. Journal of Medical Ultrasound, 16(2): 113122. 